\title{
LE MÉMOIRE DE MASTER: RUPTURES ET CONTINUITÉS. POINTS DE VUE DES ENSEIGNANTS, POINTS DE VUE DES ÉTUDIANTS
}

\section{Isabelle Delcambre* \\ Université Charles-de-Gaulle-Lille3 \\ Lille, France}

Résumé : L'écriture est une composante fondamentale de toutes les formations de l'enseignement supérieur, elle est sollicitée dans tous les dispositifs dont les objectifs se déclinent en termes d'enseignement, d'apprentissages et de formation, depuis la première année d'université jusqu'au doctorat. Au niveau du master, les étudiants et les enseignants sont confrontés à une nouvelle dimension de l'écriture universitaire: la production de connaissances scientifiques, l'insertion dans un champ de recherche. Le mémoire de master présente ainsi pour les étudiants une nouveauté par rapport aux écrits qu'ils ont pratiqués antérieurement, de nouvelles difficultés liées aux dimensions spécifiques de l'écriture de recherche en formation, et pour les enseignants, l'accompagnement de ces nouveaux écrits est liée à des attentes spécifiques, fortement liées aux différentes épistémologies disciplinaires. Cet article visera à comprendre et à analyser les enjeux de ces écrits dans la formation universitaire et la façon dont les enseignants et les étudiants les appréhendent.

Mots-clés : Littéracies universitaires. Didactiques. Disciplines. Curriculum.

Professeur Émerite en Didactique du Français. Lille3, Théodile-CIREL. Email : isabelle.delcambre@free.fr 


\section{LES LITTÉRACIES UNIVERSITAIRES : UN CADRE DIDACTIQUE}

Le champ de recherches dans lequel je m'inscris est celui des «littéracies universitaires ${ }^{1}$ », qui est proche des courants nommés Academic Literacies en Grande-Bretagne ou Composition Studies aux Etats-Unis. Ce champ de recherches, relativement nouveau en France, qui se développe cependant grâce à des manifestations et publications scientifiques (voir le colloque qui s'est tenu à Villeneuve d'Ascq en $2010^{2}$ et le numéro 153-154 de la revue Pratiques qui en est en partie issu (DELCAMBRE; LAHANIER-REUTER, 2012) n'est pas sans rapport avec ce qui se construit sous le nom de «Pédagogie universitaire ». Tout en s'en différenciant, cependant.

La pédagogie universitaire, structurée dans une association, l'AIPU (l'Association Internationale pour la Pédagogie Universitaire) qui organise de nombreuses manifestations scientifiques, n'apparait $^{3}$ en France que très récemment, comparativement aux trois ou quatre décennies d'existence de ce mouvement, qui s'est développé essentiellement au Québec et en Belgique sur le modèle des recherches anglo-saxonnes sur la formation universitaire professionnelle, par exemple dans le domaine de la santé, ou plus généralement dans ce qui s'appelle Higher Education (DE KETELE, $2010)^{4}$.

Mais ce champ apparait comme plus pédagogique que didactique : ni les questions liées aux pratiques d'écriture des étudiants (et/ou des enseignants et des chercheurs) n'y sont explicitement thématisées ni celles liées à la socialisation à des modes de pensée propres aux différentes

\footnotetext{
${ }^{1}$ Cette expression peut apparaitre comme une simple traduction de l'expression anglaise Academic Literacies, mais elle permet d'éviter les connotations dépréciatives du terme " académique » en français.

${ }^{2}$ Colloque international : Littéracies universitaires : savoirs, écrits, disciplines, organisé à l'Université de Lille 3, les 2, 3 et 4 septembre 2010 (I. Delcambre, D. Lahanier-Reuter, F. Boch, dir.). http://evenements.univ-lille3.fr/litteracies-universitaires/

${ }^{3} \mathrm{Ce}$ texte suit les recommandations officielles pour l'orthographe renouvelée du français $<$ http://www.renouvo.org/assoc.php $>$. C'est la raison pour laquelle l'accent circonflexe disparait sur un certain nombre de i et de $u$.

${ }^{4}$ L'intérêt croissant pour ces recherches en France se voit également dans la récente note de synthèse de Laure Endrizzi (ENDRIZZI, 2011).
} 
disciplines (SAUSSEZ; LOIOLA, 2008). Une analyse des publications récentes dans ce champ sur la période 1991-2005 (ADANGNIKOU, 2008) ne fait surgir qu'une seule référence à des travaux de didactique, portant sur les pratiques de l'écrit dans le supérieur, le numéro de Pratiques, (BOCH; LABORDE-MILAA; REUTER, 2004), intitulé Les écrits universitaires . Et de fait, les recherches menées sous l'étiquette de Littéracies universitaires s'inscrivent dans le champ des recherches en didactique.

La recherche qui alimente la suite de cet article s'inscrit en effet dans une perspective didactique sur l'écriture (DELCAMBRE; LAHANIERREUTER, 2011). Dans ce cadre, les pratiques d'écriture sont envisagées comme impliquées dans des enjeux d'enseignements et d'apprentissages. Cela ne signifie pas que l'on s'intéresse particulièrement à de possibles dispositifs d'enseignements ou d'apprentissages. La position didactique est d'abord théorique. Elle analyse les rôles qui constituent la situation d'enseignement et d'apprentissages comme relevant de positions différentes dans le système didactique (les étudiants vs les enseignants) et cherche ensuite à les croiser, à les confronter : que disent les étudiants des attentes des enseignants quant à leurs productions écrites ? que disent les enseignants de ces productions ? Comment définissent-ils leurs attentes? Cette perspective amène aussi à considérer les écrits et les pratiques d'écriture comme situés dans un contexte d'enseignement et d'apprentissages. Ainsi, les écrits du travail personnel (notes, écrits intermédiaires, etc.) s'opposent aux écrits qui sont destinés à être évalués par autrui: il s'agit de prendre en compte l'effet de ces différences didactiques, selon que la situation d'écriture est une situation d'évaluation (examen ou contrôle) ou une situation d'écriture privée (comme peut l'être la prise de notes), ou encore selon que l'écriture s'inscrit dans un temps long ou court.

Cette prise en compte des contextes de production des écrits oblige à différencier trois espaces de pratiques, qui peuvent caractériser les univers académiques : ce que l'on peut appeler l'écriture académique (celle à laquelle se soumettent les étudiants tout au long du cursus, pour valider leurs études), l'écriture de recherche en formation et l'écriture de recherche : cette dernière opposition est particulièrement décrite et 
théorisée par Y. Reuter (REUTER, 2004). Il montre qu'en formation, l'écriture d'un mémoire ou d'une thèse est à la fois objet et outil de formation. C'est par cette écriture que l'étudiant se forme à la recherche. Même si ces mémoires et thèses servent aussi à valider les études, ils sont une initiation à la recherche, et en ce sens cette production écrite diffère de l'écriture des chercheurs, notamment en termes de positions par rapport au savoir ou aux textes d'autrui (REUTER, 1998). Cette différence de position est à relier aux différences de discours des uns et des autres sur les mêmes objets, et amène à considérer que des discours, même opposés ou divergents, ont le même degré de pertinence au regard de ceux qui les profèrent. La prise en compte de ces positions différentes est par ailleurs un excellent garde-fou contre toute tentative normative, qui viserait à reprocher aux étudiants des manques ou des méconnaissances regrettables, par rapport aux savoirs ou aux connaissances des enseignants. Le discours des étudiants est à analyser au regard de leur position dans le système didactique.

\section{UNE RECHERCHE SUR L'ÉCRITURE À L'UNIVERSITÉ : QUESTIONS THÉORIQUES ET MÉTHODOLOGIQUES}

Cet article visera donc à comprendre et à analyser les enjeux de ces écrits dans la formation universitaire et la façon dont les enseignants et les étudiants les appréhendent. Pour ce faire, je m'appuierai sur des résultats issus de la recherche sur l'écriture à l'université que j'ai dirigée, de 2007 à $2010^{5}$. L'équipe de chercheurs s'est efforcée de construire des hypothèses de recherche en cohérence avec le point de vue didactique que j'ai exposé plus haut.

Ainsi, considérer les écritures comme didactiquement situées amène à les envisager comme indissociables des contextes où elles se déploient (et qu'elles contribuent à faire exister: sans l'écrit, bien des contextes d'apprentissage et d'enseignement universitaires n'auraient pas de sens, il

5 Recherche ANR-06-APPR-019 : «Les écrits à l'université : inventaire, pratiques, modèles ». Voir Delcambre et Boch (2011) pour un bilan global de cette recherche qui a associé didacticiens et linguistes. 
en est de même des contextes scolaires). Cependant ces contextes peuvent être définis de multiples façons: l'ancrage didactique nous a amenés à privilégier plusieurs contextes emboités les uns dans les autres. Le premier d'entre eux est le contexte disciplinaire. Les didactiques s'intéressent en effet de manière prioritaire aux contenus disciplinaires et les analysent dans leurs liens avec l'enseignement et l'apprentissage (REUTER, 2007/2010). Le poids des disciplines (d'enseignement ou de recherche) sur les pratiques d'écriture (des étudiants ou des enseignants) est la question centrale de cette recherche. Peu de chercheurs qui interrogent les pratiques universitaires se la posent. Laure Endrizzi (2011) ne signale dans sa synthèse qu'un document qui envisage le poids de l'appartenance disciplinaire : «L'empreinte disciplinaire est indéniable. Elle produit des rapports différents à la recherche et à l'enseignement ainsi que des modalités de travail très variables » (Becquet, Musselin, 2004). Certes, la question des disciplines est fort complexe à l'université (FABIANI, 2006) : parle-t-on de disciplines d'enseignement? de disciplines de recherche? Qu'est-ce qui fait l'identité des disciplines? les contenus de savoirs produits? les méthodologies mises en œuvre? les contraintes institutionnelles ? Peut-on même parler sereinement de disciplines comme si elles étaient des institutions stables et pérenne (FABIANI, 2012) ? C'est pourquoi l'équipe de recherche a décidé de s'en tenir à une définition minimale et institutionnelle, à savoir la dénomination des départements universitaires dans lesquels les étudiants ont déclaré faire leurs études. Mais nous verrons que le discours des enseignants-chercheurs met en évidence la complexité d'une appartenance disciplinaire, de leur point de vue.

Les autres contextes retenus sont ceux de l'année d'étude et du type d'institution universitaire ${ }^{6}$ dans laquelle les étudiants font leurs études. Le critère de l'année d'étude a été jugé important : il répond à l'hypothèse d'un développement des pratiques d'écriture tout au long du cursus, même si l'on peut considérer que des étudiants, parvenus à l'université, ne sont pas sans savoir écrire. Mais là encore, le point de vue didactique est important: il amène à considérer les pratiques d'écriture comme

\footnotetext{
6 Formation académique ou professionnelle, en France ou en Belgique, puisque des universités différentes étaient impliquées dans cette recherche.
} 
impliquées dans des enjeux d'enseignement et d'apprentissages qui ne sont pas forcément toujours explicités: les enjeux explicites portent le plus souvent sur des connaissances à transmettre ou à construire; mais ils supposent des pratiques de littéracie, de plus en plus savantes dans le curriculum, et qui sont souvent considérées comme des «pré-requis », ou des « allant de soi », ou encore, pour reprendre les catégories élaborées par Yves Chevallard (CHEVALLARD, 1991), des connaissances paradisciplinaires. Se demander si les discours des étudiants sur leurs pratiques d'écriture universitaire varient tout au long du cursus, et quelles sont ces variations devrait permettre de mettre en évidence s'il y a prise de conscience des implicites liés à l'écriture, ou des rapports entre construction de connaissance et mise en texte, par exemple.

Le contexte institutionnel n'était pas envisagé lors de la mise en place de la recherche, mais il est apparu comme un facteur important d'explication des différences entre certains discours d'étudiants. Nous l'avons donc retenu a posteriori.

Le lecteur a compris que cette recherche s'est principalement donné comme méthodologie le recueil de discours d'étudiants (par questionnaire et entretiens) et d'enseignants (dans des entretiens de groupes). Elle n'a pas donc étudié les textes produits par les étudiants dans la perspective de confronter leurs discours et leurs productions par exemple. Mais l'échantillon constitué a été assez large : en effet nous avons recueilli des questionnaires auprès d'environ 650 étudiants inscrits dans cinq disciplines de Sciences Humaines (Lettres Modernes, Sciences du langage, Psychologie, Sciences de l'éducation et Histoire), et cela aux cinq niveaux de la formation universitaire (trois années de Licence suivis de deux années de Master) et organisé cinq entretiens de groupes avec des enseignants titulaires, enseignant dans ces mêmes disciplines. Les étudiants étudiaient dans différentes universités (principalement en France, mais l'université libre de Bruxelles en Belgique (ULB) faisait aussi partie de nos terrains), et certains d'entre eux étaient inscrits dans un institut de formation professionnelle d'enseignants (mais je ne ferai pas état ici des résultats concernant cette population). 
Les questions de recherche interrogeaient donc le poids des disciplines sur les pratiques d'écriture déclarées: les disciplines universitaires sont-elles un facteur possible d'explication des variations enregistrées dans les déclarations des étudiants sur leurs modes d'écriture ? De même, peut-on mettre en évidence des continuités ou des ruptures dans les représentations des étudiants selon le moment où ils se trouvent dans le cursus? Enfin, quelle est l'incidence de leur appartenance institutionnelle sur ces variations de discours, en termes de types de formation (académiques générales ou professionnelles, en l'occurrence formation d'enseignants), ou d'institutions (quatre universités différentes)?

Ces trois variables permettent d'expliquer, en partie, les variations dans les discours des sujets qui seront interprétées en termes didactiques, c'est-à-dire comme liées à des variations de contextes, dont le plus intéressant, à nos yeux, est le contexte disciplinaire et épistémologique.

Concernant les enseignants, nous avons mené des entretiens de groupe avec des enseignants-chercheurs dans les cinq mêmes disciplines citées plus haut. L'objectif affiché lors de ces entretiens était de recueillir leurs discours sur leurs attentes quant à l'écriture du mémoire de master, ou pour le dire autrement, les critères d'évaluation sur lesquels ils s'appuyaient, mais aussi leurs discours sur les aides éventuelles qu'ils apportent aux étudiants, sur leurs rôles de directeur de mémoire.

Les variations (ici limitées aux variations disciplinaires ou épistémologiques et aux variations institutionnelles) permettent également d'interroger des pratiques d'accompagnement de l'écriture (ou de remédiation), soit qu'elles se présentent comme consolidation d'habilités linguistiques, soit qu'elles s'affichent comme affiliation aux genres de discours universitaires, soit encore qu'elles se réclament des pratiques d'écriture scientifique qui sont les leurs.

\section{LE MÉMOIRE : PREMIĖRE APPROCHE}

Cette première approche tente de définir comment les étudiants conçoivent le mémoire, genre qui apparait au niveau du master et qui, souvent, n'est pas précédé par des expériences d'écriture longue similaire. 
L'analyse de leurs discours sur les écrits qu'ils ont à produire à l'université selon la variable du niveau d'étude met en évidence des clivages importants entre la licence et le master, et parfois des clivages à l'intérieur même de chaque cursus (la licence dont il est question ici dure trois ans et le master, deux ans). J'exposerai également quelques résultats à propos des normes que les étudiants mettent en œuvre dans la production d'un mémoire, ainsi que des normes qu'ils pensent être celles des enseignants évaluateurs, et pour finir, je donnerai quelques aperçus de leurs discours sur les difficultés rencontrées dans les différents genres de discours auxquels ils sont confrontés dans leurs études.

\subsection{SPÉCIFICITÉS DU MÉMOIRE}

Le mémoire apparait massivement dans le discours des étudiants comme un nouveau genre discursif, caractéristique de la formation en Master. Il distingue nettement les déclarations recueillies en Master par rapport à celles recueillies en Licence, du moins dans les universités françaises. En Belgique, en effet, à l'ULB, les étudiants ont, dès la seconde année du Baccalauréat (équivalent belge de la licence française), des " travaux de séminaires » à réaliser qui sont des initiations à une écriture longue qui suppose 1'élaboration d'une question, un recueil et un traitement de données. Par exemple, dans le séminaire d'histoire du Moyen Age en $3^{\circ}$ année de Baccalauréat, 1'évaluation porte sur 1'«Elaboration d'un travail écrit de recherche sur une localité durant le Moyen Âge et à l'époque moderne. ». Dans certaines disciplines, en France aussi, dès la $3^{\circ}$ année de licence, les étudiants ont à produire des travaux d'étude (par exemple, en Psychologie à l'université de Lille3) ou des dossiers d'observation (en Sciences de l'Education, à Lille3, toujours). Ce «mémoire de Licence » peut être vu comme une des conséquences de l'espace européen d'enseignement supérieur, tel qu'il s'est construit suite aux accords de Bologne, mais il est très diversement représenté selon les pays et les cultures. En tout cas, lorsque les étudiants précisent quel est l'écrit représentatif de leur année de formation dans la discipline où ils sont inscrits, la dénomination « mémoire » n'apparait qu'au niveau du Master. 


\subsection{QUELS SONT LES ÉCRITS LES PLUS FRÉQUENTS EN LICENCE ET EN MASTER SELON LES ÉTUDIANTS ?}

Dans leurs réponses à la question sur l'écrit qu'ils jugent représentatif de leurs études, apparait clairement un contraste entre l'unicité de l'écrit qui caractérise le master et dénote l'entrée dans la recherche, et la multiplicité des écrits pratiqués en licence: exposés, travaux d'études, travaux de séminaire, compte-rendu, synthèses, dissertations, Questions à choix multiples (QCM), rapports de stage, commentaires, dossiers, résumés, etc. (voir annexe 1).

On peut interpréter ce contraste comme l'indication d'un trajet tout au long du cursus, trajet d'une hétérogénéité des pratiques instituées vers une homogénéité, qui ne doit cependant pas tromper, car l'homogénéité du discours des étudiants en Master masque la grande diversité des types de mémoires selon les disciplines et les méthodologies.

Ce trajet se voit aussi au sein de la licence : entre les deux premières années et la troisième année, les étudiants ne sont pas confrontés aux mêmes types d'écrits : résumés, dossiers, commentaires, fiches, rapports de stage caractérisent, selon les étudiants, les deux premières années; dissertations, synthèses, compte-rendu, travaux de séminaire, QCM, exposés, Travaux d'études, sont plutôt des écrits de troisième année.

En tout état de cause, il y a bien des trajets dont rendent compte ces discours des étudiants et les confrontations avec de nouveaux genres discursifs, tout au long du cursus, peuvent être une des causes des difficultés ressenties (DELCAMBRE; LAHANIER-REUTER, 2012).

\subsection{QUE DISENT LES ÉTUDIANTS DE LEURS NORMES PERSONNELLES?}

Le questionnaire interrogeait les étudiants sur les normes qu'ils mobilisent lorsqu'ils écrivent, c'est-à-dire sur ce à quoi ils font particulièrement attention lorsqu'ils produisent cet écrit représentatif de leurs études (cf. Annexe 2). A partir des items choisis et hiérarchisés par les 
étudiants, nous avons pu construire quatre profils différents ${ }^{7}$. L'un d'entre eux différencie nettement les étudiants de master par rapport aux autres, c'est celui que nous avons appelé « ceux qui écrivent avec autrui, à partir de textes d'autrui ». En ce qui concerne la licence, de nouveau la répartition des réponses selon les profils fait apparaitre un clivage entre les étudiants de première année et les autres : les premiers sont majoritaires à entrer dans les profils de « ceux qui recherchent la réponse correcte» ou de « ceux pour qui privilégient l'expression personnelle dans leur écriture ». Les autres sont ceux qui le plus «cherchent à écrire clairement et correctement $»$.

Ainsi les étudiants de Master découvrent dans l'écriture du mémoire, les questions de l'intertextualité et de l'interdiscursivité (DELCAMBRE, 2012); questions absentes des préoccupations des étudiants des années antérieures.

\subsection{QUE DISENT LES ÉTUDIANTS DES ATTENTES DES ENSEIGNANTS?}

Parallèlement aux questions sur les normes personnelles, le questionnaire interrogeait les étudiants sur leurs représentations des normes des enseignants, notamment sur ce que, d'après eux, les enseignants privilégient dans leurs évaluations de l'écrit dit représentatif.

De nouveau, les choix des étudiants sont nettement différenciés par leur année d'étude. Le facteur le plus important est celui de la différence entre Licence et Master, mais on retrouve, comme précédemment un trajet de la première à la cinquième année d'université. C'est peut-être un des trajets les plus spectaculaires mis au jour dans cette recherche (annexe 3).

Pour commencer, les étudiants de licence sont plus nombreux à dire que les enseignants privilégient la justesse de la réponse, la correction de la langue, l'orthographe, alors que les étudiants de master pensent que les enseignants privilégient la qualité des recherches, l'originalité, la compréhension des textes lus ainsi que les articulations et la cohérence globale de leur écrit.

\footnotetext{
${ }^{7}$ Ces profils ont été construits à partir d'une analyse factorielle (analyse en composantes principales) des différents regroupements des 13 items proposés aux étudiants. Ils peuvent devenir la base des comparaisons entre disciplines ou niveaux d'étude.
} 
Un deuxième facteur de différence est celui qui distingue la première année d'un cursus (L1, M1) des années suivantes (respectivement L2, L3 et M2). En conséquence, on peut décrire quatre groupes de réponses, déterminées par ces années :

- Les étudiants en première année de licence ne s'éloignent pas des critères de surface (orthographe, style) dans leur appréciation des normes évaluatives des enseignants

- Les étudiants de $2^{\circ}$ et $3^{\circ}$ année de licence s'emparent de critères plus disciplinaires (les sources) et privilégient outre la clarté du discours et la pertinence des réponses, la qualité des reformulations

- Les étudiants en première année de Master découvrent des écrits dont ils ont à élaborer la problématique, dont ils sont les auteurs, mais qu'ils composent à partir de lectures difficiles

- Les étudiants de seconde année de master retiennent les articulations (entre parties d'un texte, mais aussi entre concepts et théories), les références bibliographiques, l'argumentation, bref, des critères que l'on verrait bien proches de ceux des écrits scientifiques.

Ce trajet, disais-je plus haut, est un des plus intéressants, entre autres parce qu'il donne à voir le passage de normes linguistiques au début de la licence à des normes discursives et scientifiques à la fin du master. Loin d'être l'indice de ruptures, il me semble qu'il illustre parfaitement une évolution de la représentation des attentes universitaires concernant la production d'écrits chez les étudiants qui ont été interrogés dans cette recherche (DELCAMBRE; LAHANIER-REUTER, 2011).

\subsection{QUELLES DIFFICULTÉS LES ÉTUDIANTS DÉCLARENT-ILS RENCONTRER SELON LES DIFFÉRENTS GENRES D'ÉCRITS ?}

Quelques questions ouvertes portaient sur les difficultés (ou les facilités) rencontrées et demandaient aux étudiants de produire des réponses développées, que nous avons traitées par la méthode de l'analyse de contenu en mettant en évidence des thèmes dominants et des sous-thèmes (DAUNAY; LAHANIER-REUTER, à par.). 
Quatre thèmes seulement, décrivant les difficultés rencontrées, organisent ou séparent les genres de discours ${ }^{8}$ (voir annexe 4) :

- Ce à partir de quoi on écrit, les matériaux de l'écriture

- l'intertextualité (les lectures et les auteurs)

- La cohérence, notamment du plan

- les contraintes, en particulier de temps

Les difficultés particulières au mémoire au niveau du master sont principalement ce à partir de quoi les étudiants écrivent (les données à rassembler, les recherches bibliographiques, etc.) et curieusement relativement peu les difficultés qui seraient liées à la lecture et au positionnement par rapport aux auteurs (qui sont davantage liées aux pratiques de commentaires, d'analyses et de synthèses de textes), alors que cette dimension de l'écriture avait été signalée comme importante voire primordiale dans les normes personnelles des étudiants au niveau master. Cette distorsion signifie peut-être que la dimension intertextuelle de l'écriture de recherche ne leur pose pas tellement problème (à la différence de ce que pourraient peut-être en penser des enseignants !).

En bref, le mémoire, malgré l'ambigüité de ce que recouvre ce terme, qu'il faut postuler très différent selon les méthodologies et les épistémologies disciplinaires, est clairement identifié par les étudiants comme LA spécificité du master, nouveauté tellement nouvelle qu'elle est liée, dans l'esprit des étudiants, à des difficultés spécifiques, liées au travail préparatoire à l'écriture et à une focalisation particulière sur la dimension interdiscursive de l'écriture. Quant à leurs représentations des attentes particulières des enseignants sur ce genre de discours, on a vu qu'elles étaient particulièrement précises et décrivaient, tout au long des deux années du cursus, un affinement de la perception de ce que peut être un écrit de recherche en formation (REUTER, 2004).

\footnotetext{
${ }^{8}$ Ce sont de nouveaux thèmes par rapport à ceux des graphes précédents: ils sont en effet issus des réponses des étudiants et non des items du questionnaire
} 


\section{LA VARIABLE « DISCIPLINE » : LES CONTENUS CONVOQUÉS DANS LES MÉMOIRES}

J'évoquais en introduction de cet article les difficultés posées par la notion de discipline universitaire. Cependant, c'est une dimension incontournable de l'analyse des pratiques universitaires d'écriture, notamment au niveau du master. En effet, les mémoires manifestent, dans leurs modalités d'écriture, dans leur organisation discursive, dans leurs rapports aux sources, etc., les dimensions épistémologiques des disciplines dans lesquelles ils sont produits. Par ailleurs, un des paradoxes de l'écriture et du système des genres discursifs est qu'ils produisent tout autant les traditions disciplinaires qu'ils en sont le produit. Les liens entre écriture et épistémologie sont particulièrement étroits (DAUNAY, 2008) et l'écriture d'un mémoire de master est l'occasion de les découvrir et de les éprouver.

Cependant, Y. Reuter a bien montré que les pratiques d'écriture en master ou en doctorat ne peuvent pas être considérées tout à fait de la même nature que les pratiques d'écriture des chercheurs. Il propose ainsi (REUTER, 2004) de distinguer l'écriture de recherche de l'écriture de recherche en formation. Les analyses linguistiques des écrits scientifiques montrent en effet que les pratiques ne sont pas tout à fait les mêmes, que les normes ne sont pas tout à fait respectées de la même manière par les chercheurs patentés et par les «novices» (voir, entre autres, $\mathrm{BOCH}$ (2008)).

\subsection{SPÉCIFICITÉS DES DISCIPLINES}

Je veux redire cependant la difficulté de la notion de discipline à l'université: en effet, parle-t-on d'une discipline d'enseignement ou d'une discipline de recherche ? Et dans ce dernier cas, la notion de discipline estelle viable, tant les contenus et les méthodes créent des espaces différents à l'intérieur de ce qu'on appelle «discipline» dans les programmes d'enseignement? Ces espaces sont particulièrement visibles dans l'éclatement des disciplines d'enseignement au niveau du master: les masters décomposent/recomposent les champs disciplinaires abordés en 
Licence. Par exemple, une discipline comme la psychologie qui se manifeste au niveau Licence ${ }^{9}$ sous une seule appellation (licence de psychologie) éclate au niveau master en huit spécialités au niveau master ${ }^{10}$. Il en est de même en Sciences du langage : après une " licence de Sciences du langage $»$, les étudiants se voient proposer quatre spécialités de master ${ }^{11}$, etc.

Nous voyons des traces de ces spécificités épistémologiques dans les discours tenus par les enseignants dans les entretiens de groupe, lorsqu'ils sont en discussion sur leurs exigences et leurs attentes par rapport aux mémoires de master. Observons ces deux extraits de l'entretien mené à Bruxelles avec des chercheurs en Histoire :

«alors la réponse de $\mathrm{V}$. sur la mise en contexte par rapport à l'actualité est très symptomatique de l'approche à l'intérieur de la discipline, parce qu'elle est historienne de l'époque contemporaine, et donc il y a sans doute une attente plus forte [sur cette spécificité] » (MG, 53)

«j'ai quand même vu, en histoire, des notes [d'examen] qui allaient du simple au double, c'est peut-être chez nous...enfin en histoire contemporaine... mais on voit vraiment une différence, les attentes sont différentes » $(\mathrm{VP}, 126)$

Les enseignants verbalisent ici des attentes sur un contenu spécifique, discursif et épistémique (contextualiser la question de recherche du mémoire par rapport à l'actualité) ou des pratiques d'évaluation qu'ils relient fortement à un champ de recherche particulier à l'intérieur de la discipline « Histoire », celui de l'histoire contemporaine.

\footnotetext{
${ }^{9}$ L'exemple est celui du cursus de psychologie de l'université de Lille3.

10 Psychopathologie et psychologie clinique; Neuropsychologie clinique; Analyse expérimentale et appliquée du comportement ; Psychologie du travail et des organisations ; Psychologie du travail, option EPPSY ; Psychologie Clinique des interventions cognitivocomportementales, Educatives et Sociales; Psychologie des processus neurocognitifs et sciences affectives ; Psychologie clinique et sociale de la santé.

${ }^{11}$ Français Langue Etrangère ; Langue des Signes Française ; Lexicologie, terminographie, traitement automatique des corpus ; Linguistique.
} 
Autre exemple, issu de l'entretien avec des chercheurs en psychologie à Lille. Ces entretiens de groupe amenaient les chercheurs à commenter deux bons mémoires, produits dans leur « discipline », dans une autre université, pour articuler leurs avis et leurs attentes à des exemples précis, et éviter les discours trop généraux ou de déploration sur les incompétences des étudiants. Dans ces trois extraits, c'est la même personne qui s'exprime, en commentant donc les deux mémoires, intitulés Sensibilité à la morphologie lexicale et Apprentissage de la lecture et Implication des aspects verbaux de la mémoire et du centre exécutif en lecture chez des enfants de CE1.

«Ben globalement, bon, ce sont deux champs de recherche qui sont très éloignés du mien mais globalement c'est vrai quand je les ai parcourus, déjà je sais que c'est des bons mémoires » (M 26)

«moi j'ai un problème avec le langage et donc je ne comprends jamais rien (rires) au contexte théorique. Mais je pense que c'est un blocage, donc je ne vais pas aller plus loin là-dedans. Mais je comprends jamais rien ». (M 33)

«moi j'ai jamais eu de cours de langage, à Grenoble il n'y avait aucun spécialiste du langage. Donc je n'ai jamais eu de cours de langage. » (M 46)

Nous voyons ici un exemple classique d'aveu d'incompétence : le chercheur non spécialiste d'un champ, d'une question, d'un terrain, d'une méthodologie, etc., se refuse à juger ce qu'il ne connait pas. Ici, quelles qu'en soient les raisons, «le langage », tout important qu'il est en psychologie, est un domaine que cet enseignant ne maitrise pas et sur lequel il refuse de se prononcer, sans que cela entame sa légitimité, au contraire (voir les rires).

Ainsi, pour revenir aux étudiants, un étudiant de master en psychologie ne sera pas aux prises, bien évidemment, avec les mêmes cadres théoriques et méthodologiques, ni même avec les mêmes normes d'écriture, en psychologie expérimentale, en psychologie clinique, en psychologie sociale, en psychologie développementale; en Sciences du 
langage, de même, il ne sera pas confronté aux mêmes attentes scientifiques s'il s'inscrit en didactique du Français Langue Etrangère, en lexicologie ou en traitement automatique de corpus, etc. Les contenus disciplinaires sont à ce niveau fortement liés à des méthodes et à des questions de recherche spécifiques ou à des terrains particuliers (la santé, les troubles des fonctions psychiques supérieures (langage, mémoire), le travail, le développement, etc.) ou encore à des « champs de savoirs » renvoyant à des dimensions différentes de la langue: lexicographie, syntaxe, sémantique, etc.

\subsection{PEUT-ON IDENTIFIER UN DISCOURS DES ÉTUDIANTS SUR LES DIMENSIONS ÉPISTÉMOLOGIQUES DE L'ÉCRITURE DU MÉMOIRE?}

Ces discours ne sont pas faciles à identifier, car les étudiants n'étaient pas interrogés explicitement sur ces questions. Cependant, on peut repérer a posteriori des traces de cette dimension disciplinaire de l'écriture en traitant les réponses aux questions sous l'angle des variations induites par les disciplines (et non plus du point de vue des variations liées à la position de l'étudiant dans le cursus, comme je l'ai fait en première partie de cet article). Méthodologiquement, on ne peut que reconstruire à travers leurs réponses au questionnaire, un discours second des étudiants sur les contenus: ainsi, ce qu'ils disent de leurs difficultés peut révéler en creux les contenus visés, mais non atteints; ce qu'ils disent des attentes des enseignants est peut-être plus direct, mais cette reconstruction des attentes doit être comprise comme très générale, car l'étude selon la variable « discipline » mêle tous les niveaux.

Dans la suite, j'exposerai donc ce que la variable « discipline » nous apprend sur les normes que les étudiants disent appliquer aux genres d'écrits qu'ils produisent et sur les attentes qu'ils disent être celles des enseignants (les normes d'évaluation). Je présenterai pour finir quelques résultats sur les difficultés déclarées: quels sont les écrits difficiles et quelle est la nature des difficultés rencontrées? 


\subsection{LES NORMES PERSONNELLES SE DIFFÉRENCIENT-ELLES SELON LES DISCIPLINES ?}

L'appartenance disciplinaire des étudiants, tout comme leur année d'études, produit des répartitions intéressantes entre les profils déjà utilisés ci-dessus pour l'analyse des normes personnelles (cf. annexe 5).

$\mathrm{Si}$ tous les étudiants partagent l'idée qu'il faut mobiliser ses connaissances et trouver la réponse juste (profil "répondre correctement"), les trois autres profils se répartissent clairement selon les disciplines, sauf en ce qui concerne l'Histoire (à Lille comme à Bruxelles, les étudiants ne peuvent pas être caractérisés nettement par l'un de ces profils). On voit une nette opposition entre les étudiants de Psychologie et de Sciences de l'Éducation qui disent surtout "écrire à partir de textes" (c'est-à-dire toutes les dimensions interdiscursives de l'écriture universitaire: reformuler, discuter les auteurs, faire des références et des citations) et ceux de Lettres Modernes et de Sciences du langage, plus centrés sur les aspects transversaux de l'écriture, non spécifiquement scientifique ou universitaire: les uns disent faire particulièrement attention au style, à l'originalité et à la formulation d'un avis personnel (ce que nous avons appelé le profil "expression de soi“) ; les autres déclarent faire attention à "écrire clairement" (c'est-à-dire veiller à la clarté formelle, à la correction de la langue, aux articulations et à la clarté du discours en général).

\subsection{LES ATTENTES SUPPOSÉES DES ENSEIGNANTS SONT-ELLES DIFFÉRENCIÉES SELON LES DISCIPLINES ?}

Je rappelle qu'il s'agit ici des critères d'évaluation considérés par les étudiants comme privilégiés par les correcteurs (annexe 6).

Comme précédemment, une discipline, ici les Sciences de l'éducation, échappe à la particularisation : dans ce cursus, les étudiants ont du mal à identifier les attentes de leurs enseignants ou à les caractériser. Par contre, une formation se distingue très nettement des autres: l'Histoire, telle qu'elle est enseignée à Bruxelles. En effet, les étudiants de ce cursus déclarent que leurs enseignants privilégient les sources et les références 
bibliographiques dans leur évaluation, alors que dans les autres cursus (tous français), les déclarations des étudiants partagent 1'Histoire et les Sciences du langage, d'un côté, et de l'autre, la Psychologie et les Lettres modernes. Pour les premiers, les attentes des enseignants portent surtout sur l'orthographe, la correction de la langue, le style, l'avis personnel, pour les seconds, sur la problématique, l'argumentation, le contenu et son organisation.

On voit que, comme précédemment, il n'y a pas toujours concordance entre les normes personnelles des étudiants et celles qu'ils attribuent aux enseignants : cela peut se comprendre, notamment si l'on prend en compte les positions dans le système didactique. Ce à quoi les étudiants disent faire attention lorsqu'ils écrivent peut être interprété comme des contenus qu'ils auraient intériorisés, ce à quoi les enseignants font attention d'après eux serait plutôt à interpréter comme des contenus imposés de l'extérieur, à acquérir probablement. Les discordances entre ces deux zones de représentations pourraient être analysées comme la manifestation d'un programme d'apprentissage.

Ainsi, en Lettres modernes, si les étudiants (toutes années confondues) disent faire attention à l'expression, ils savent que leurs enseignants vont se centrer sur la problématique et l'argumentation. Seraitce une discipline dont les spécificités seraient vécues comme contradictoires par les étudiants, ou dont ils percevraient les spécificités discursives comme un horizon encore lointain?

Deux autres disciplines apparaissent comme éclatées dans les représentations des étudiants : Les étudiants de Sciences de l'Education ont une idée de ce à quoi ils font attention quand ils écrivent (l'interdiscursivité), mais ne savent pas se prononcer sur les attentes des enseignants. Et à l'inverse, les étudiants d'Histoire, ceux de Bruxelles, s'ils ne se distinguent pas à propos des normes personnelles (ils mettent l'accent comme tous les étudiants sur l'importance de répondre correctement), ont une idée très claire de ce qu'attendent leurs enseignants: faire des références bibliographiques! Ces étudiants se distinguent souvent des autres (y compris de leurs homologues français : voir ci-dessous) : non seulement dans le choix unanime de l'écrit représentatif de leur formation 
(les travaux de séminaire), mais aussi dans celui des critères d'évaluation de leurs enseignants. Rappelons que la spécificité de ce cursus, notamment par rapport aux études historiennes françaises, est assumée voire revendiquée par les enseignants dans les entretiens de groupe (voir plus loin), mais que les critères d'évaluation ici cités (la qualité des sources et celle des références bibliographiques) sont ceux que ces enseignants avancent et pensent être partagés par tous les historiens.

Pour finir, Sciences du langage et Psychologie apparaissent comme des disciplines plus homogènes du point de vue des normes: dans la première, on voit une cohérence autour de la clarté du discours et de la correction de la langue, dans la seconde, sur l'interdiscursivité, la problématique et l'argumentation. Est-on là en présence de deux épistémologies «spontanées »? En tout cas, les étudiants de ces cursus montrent une certaine unité entre ce qu'ils se donnent comme normes et ce qu'ils identifient comme normes externes à travers les pratiques d'évaluation des enseignants.

\subsection{QUELS ÉCRITS SONT DÉCLARÉS DIFFICILES SELON LES DISCIPLINES ?}

Pour approfondir ces questions, nous pouvons interroger les difficultés déclarées par les étudiants. Et tout d'abord, la variable « discipline » différencie-t-elle les écrits dans lesquels ils disent rencontrer des difficultés?

Il s'agit, sans surprise, des écrits déclarés comme les plus représentatifs de chacune des disciplines ${ }^{12}$, avec une nette partition: la formation en Histoire à Bruxelles est opposée à celles en Histoire à Lille et en Lettres modernes. Les étudiants bruxellois ont à écrire des travaux de séminaire ou des écrits d'examen, les étudiants français, des dissertations. Et ces écrits sont aussi ceux qui leur posent problème.

Dans les autres disciplines, Sciences du Langage, Sciences de l'éducation et Psychologie, les écrits difficiles sont, respectivement, des

\footnotetext{
${ }^{12}$ Un des résultats majeurs de cette recherche est de montrer que chaque discipline, chaque département est spécifié par des écrits différents (DELCAMBRE ; LAHANIER-REUTER, 2009).
} 
dossiers ou des rapports de stage, des mémoires ou des travaux d'étude et de recherche (TER), des mémoires, des TER ou des synthèses. On voit la dispersion des types d'écrits différents, dans ces disciplines qui ne sont pas dans la continuité avec des disciplines scolaires, et qui confrontent donc les étudiants avec des types d'écrits entièrement nouveaux, ou presque.

Cela dit, que les écrits soient nouveaux ou déjà connus des étudiants comme la dissertation qui est un exercice phare du baccalauréat français dans de nombreuses disciplines diferentes (DENIZOT; MABILONBONFILS, 2012), ils sont identifiés comme posant des difficultés.

\subsection{QUELLES DIFFICULTÉS SONT-ELLES DÉCLARÉES PAR LES ÉTUDIANTS EN FONCTION DES DISCIPLINES ?}

Comme on l'a déjà vu plusieurs fois antérieurement, une discipline apparait comme non spécifiée en tant que groupe par ces thèmes : c'est la Psychologie.

Par contre, on remarque (annexe 7) une opposition principale entre les étudiants de Lettres modernes et ceux de Sciences du Langage: pour les premiers, c'est plutôt la nouveauté de l'écrit qui leur pose problème (ce qui est curieux quand on sait qu'ils écrivent surtout des dissertations, genre discursif pratiqué dès le lycée) ; les seconds disent rencontrer des difficultés avec le discours, c'est-à-dire la clarté de l'exposition, et les lectures, difficiles à comprendre, mais aussi avec le recueil des données, antérieur à l'écriture de leurs dossiers ou rapports de stage.

Les étudiants en Histoire à Bruxelles montrent de nouveau une position très particulière, en déclarant avoir des difficultés à cerner les attentes des enseignants (ce que nous avons appelé « difficultés avec les situations de production »). Les étudiants des autres disciplines signalent avoir du mal à convoquer les auteurs et à ne pas faire de paraphrase.

On le voit, les résultats produits par l'étude du corpus des réponses des étudiants grâce à la variable Discipline sont moins lisibles, moins systématiques, ils concourent à former une vue d'ensemble moins nette que ceux produits par la variable « Année d'étude ». Nous en avons conclu que la conscience disciplinaire des étudiants (REUTER, 2007) est inégalement 
construite, claire sur certains aspects, floue sur d'autres (mais cela peut être dû au traitement des réponses : ces résultats sont obtenus en globalisant toutes les réponses de la première à la cinquième année d'études). En tout état de cause, leurs déclarations, filtrées par la question disciplinaire, ne permettent pas de dessiner un contour net des représentations qu'ils se font des épistémologies disciplinaires dans lesquelles ils sont pourtant plongés.

\section{7 ÉLÉMENTS DE CONCLUSION SUR LES CONTENUS DISCIPLINAIRES DU POINT DE VUE DES ÉTUDIANTS}

Je propose cependant, en guise de conclusion de cette deuxième partie, de prendre deux disciplines qui peuvent faire exemple, par leurs éléments de cohérence.

Le premier exemple est celui des Lettres Modernes : les déclarations des étudiants permettent d'identifier une forme de cohérence disciplinaire, notamment dans le lien qu'ils font entre la dissertation (comme écrit représentatif et écrit difficile) et les normes personnelles de style, d'originalité et d'expression d'une opinion personnelle. Certes, on peut discuter de cette image de la dissertation, tous ne la partagent pas, mais elle est attestée par ailleurs (DELFORCE, 1985). Cependant, la distorsion relevée ci-dessus entre ces normes personnelles et les attentes des enseignants (qui portent sur la qualité de la problématique, de l'argumentation ou de l'organisation du contenu) sont signes d'une évolution possible vers un autre modèle de la dissertation, non encore intériorisé par les étudiants.

Le second exemple est celui de l'histoire et des oppositions frontales entre ce qu'en disent les étudiants bruxellois et les étudiants français de Lille. Les étudiants bruxellois sont très conscients du rôle dans l'écriture historique de l'établissement des sources, de l'importance des références bibliographiques et pratiquent un genre d'écrit dont la désignation leur est spécifique, celle des travaux de séminaire, qu'ils pratiquent dès la Licence. Les étudiants en Histoire à Lille, quant à eux, sont plus proches dans leurs déclarations des étudiants de Lettres modernes français que de ces étudiants bruxellois, qu'il s'agisse des genres spécifiques à leurs études, les dissertations et les commentaires de textes ou de l'identification des normes 
d'évaluation, l'orthographe, la correction de la langue et le style. On peut voir dans ces pratiques historiennes différentes l'influence des concours de recrutement pour l'enseignement secondaire en France et la toute-puissance de la dissertation dans ces concours qui donnent forme à l'enseignement universitaire, et qui ont plus de poids dans les représentations des étudiants que les pratiques de la recherche historienne et des genres de discours qui l'accompagnent. Nous avons là une opposition épistémologique claire, liée aux traditions d'enseignement et de formation, qui nous ont fait prendre en compte le fait qu'une même discipline, outre les partitions internes qu'elle connait de par les objets d'étude (voir par exemple, le cas de l'histoire contemporaine, cité plus haut), peut être soumise à des variations sensibles d'une institution à une autre.

\section{FRAGMENTS DE DISCOURS DES ENSEIGNANTS}

Dans le cadre de la recherche qui a permis d'établir ces résultats, nous avons mené des entretiens de groupe avec des enseignants-chercheurs dans les cinq disciplines dans lesquelles nous avons interrogés les étudiants. Le dispositif mis en œuvre a consisté à réunir pendant 2 heures 3 à 7 enseignants dans chaque discipline (la variation de ce nombre est tout à fait contingente et liée aux disponibilités des enseignants). L'entretien était mené par un chercheur extérieur à l'institution (pour éviter des effets de conformation ou la réactivation de tensions internes) à partir d'un guide d'entretien (pour assurer la comparabilité des discours d'un groupe à un autre) et de deux extraits de mémoire de master $1^{\text {ère }}$ année qui avaient été fournis aux interviewés auparavant, afin que les discours puissent s'ancrer dans des exemples concrets (il s'agissait de la page de titre anonymée, du sommaire, de l'introduction et de la conclusion). Ces mémoires avait été produits dans une autre université et avaient été jugés bons, voire très bons, dans l'institution d'où ils provenaient. L'objectif affiché était celui de recueillir les discours des enseignants-chercheurs sur leurs attentes quant à l'écriture du mémoire de master, ou pour le dire autrement, les critères d'évaluation sur lesquels ils s'appuyaient, mais aussi sur les aides éventuelles qu'ils apportaient, sur leurs rôles de directeur de mémoire (LAHANIER-REUTER; DELCAMBRE, 2013). 
Après avoir présenté des éléments d'analyse du discours des étudiants, je vais donc m'attacher dans ce qui suit à présenter comment les enseignants-chercheurs définissent le genre discursif du mémoire, quelles sont leurs attentes quant au travail que doivent fournir les étudiants. Nous verrons aussi s'ils font des liens avec leurs spécialités disciplinaires ou avec leurs propres pratiques d'écriture scientifique.

\subsection{LE MÉMOIRE : VERS UNE DÉFINITION?}

Dans le discours des enseignants sur le mémoire, la première chose à remarquer est qu'ils ne le définissent pas, qu'ils ne précisent pas les caractéristiques du genre (LAHANIER-REUTER ; DELCAMBRE, 2013). Deux explications à cela : les enseignants n'ont pas besoin de définir ce qu'ils entendent par un mémoire de master, ils fonctionnent à l'implicite, ils appartiennent à la même institution, à la même discipline, cela va de soi pour eux ${ }^{13}$. Ou bien, ils n'ont pas l'occasion de se rencontrer pour se poser ce genre de question: ainsi, en Histoire à Bruxelles, l'un des enseignants déclare : «c'est rare que nous ayons l'occasion de se mettre autour de la table pour parler de ce qu'on attend des étudiants ».

\subsection{CE QU'IL N'EST PAS}

S'il est défini, c'est par opposition à ce qu'il n'est pas. Par exemple, les enseignants de Lettres Modernes, l'opposent à l'essai; ceux des Sciences du Langage, au rapport de stage, ceux des Sciences de l'Education, à la fiche de lecture ; ceux d'Histoire (à Bruxelles ${ }^{14}$ ) soit à l'essai anglo-saxon soit à la dissertation «à la française ». Ces oppositions dessinent en creux le mémoire, de manière implicite, donc.

Le mémoire trouve ainsi ses spécificités implicites dans cette délimitation par rapport à d'autres genres académiques (qu'ils soient

\footnotetext{
${ }^{13}$ Un contre-exemple intéressant s'observe en Sciences de l'éducation où la diversité des «sous-disciplines » constitutives de cette discipline académique peut expliquer qu'un enseignant éprouve le besoin de le définir à un moment de l'entretien de groupe.

${ }^{14}$ Les enseignants d'Histoire français n'ont pas été interviewés.
} 
européens ou anglo-saxons). Le discours des historiens belges est particulièrement intéressant, par ses dimensions liées à une pratique d'enseignement et à une tradition institutionnelle différentes qui permettent de bien mettre en évidence ce qui, sans cela, resterait plus ou moins caché dans l'évidence de la tradition qui est celle du chercheur.

«La formation d'historien en Belgique est beaucoup plus libérée de la formation littéraire et scolastique qu'en France et c'est ça qui fait la différence (JPD, 77)

Pour ces historiens belges, la formation française est essentiellement liée à la dissertation :

« Il y a des différences aussi de formation en histoire en France et en Belgique, c'est-à-dire que nous n'avons pas de dissertations historiques par exemple, donc les historiens français mettent l'accent sur l'écriture de l'histoire alors que nous, notre formation met l'accent sur la recherche (VP, 40)

«[les étudiants] n'ont pas le training de la dissertation, et donc quand ils ont eu la chance de faire de la dissertation dans le secondaire, ils l'ont en général oublié, de telle sorte que quand ils sont devant une dissertation, ils sont incapables de reconnaître à quel genre littéraire ça correspond, et ça c'est une force et une faiblesse de la formation d'un historien en Belgique, c'est qu'on a l'impression que les étudiants en France sont obsédés par l'idée de respecter la forme d'écriture qu'on leur demande, mais du coup ils ont le bénéfice de la clarté de raisonnement et ils savent très bien ce qu'est une question de recherche, ils ont l'habitude de la poser, puis d'y revenir en conclusion, tandis que chez nous, si on donne un travail aux étudiants et qu'on ne leur donne pas un plan du travail avec une méthodologie, en disant à tel endroit vous devez mettre telle chose... ils remettent parfois quelque chose qui est génial, où ils ont découvert quelque chose, mais ce n'est pas une démonstration » (JPD, 68)

Le discours est ambigu et complexe («force et faiblesse ») : la dissertation est perçue comme le parangon «d'une formation littéraire et scholastique », d'une formation qui met plus l'accent sur l'écriture que sur 
la recherche, mais aussi d'une formation au raisonnement ou à la démonstration. D'où la prise de distance, mais aussi le regret que la dissertation n'irrigue pas davantage les pratiques d'écriture des étudiants belges.

L'intérêt de ces réflexions un peu périphériques à notre propos est cependant de mettre le doigt de nouveau sur les liens épistémologiques entre formation, recherche et écriture, l'écriture étant vue ici non sous les dimensions linguistiques transversales (l'orthographe, la syntaxe, le lexique, etc.) mais sous les aspects du genre discursif et de ce que le genre impose comme contraintes de pensée aux scripteurs : poser une question de recherche, la reprendre en conclusion, faire une démonstration.

\subsection{CE DONT IL EST PROCHE}

Dans la même perspective, définir le genre du mémoire par comparaison avec d'autres genres proches est une démarche possible, mais rare, que nous n'avons trouvée qu'une fois, en Sciences de l'éducation. L'enseignant réfléchit ici sur les proximités entre le mémoire (nommé ici « travail d'étude et de recherche », TER) et la fiche de lecture.

«les étudiants produisent un travail d'étude et de recherche théorique, c'est à dire plutôt centré sur l'élaboration de questions de recherche ou de problématiques autour de lectures théoriques, donc c'est la fiche de lecture un peu plus élaborée avec mise en dialogue des auteurs, mise en évidence des convergences, des divergences, enfin, une lecture intelligente de recherches avec des auteurs » (Sciences de l'éducation).

On voit comment cette comparaison amène l'enseignant à préciser les spécificités de la fiche de lecture lorsqu'elle devient une dimension du mémoire, dans sa partie théorique : faire dialoguer les auteurs, s'initier à des formes de synthèse (convergences et divergences) qui devraient aboutir à l'élaboration d'une problématique à partir des textes de recherche ainsi interrogés et discutés. 


\subsection{CE QU'IL N'EST PLUS}

« Nous ne faisons plus de mémoire ici [...] on a essayé de préserver cette forme du mémoire unique mais maintenant on a TER théorique, TER empirique, donc ce sont deux choses séparées et on n'a pas forcément [...] quelque chose qui ressemble à un mémoire de maîtrise » (Sciences de l'éducation).

Cette réflexion qui prend en compte l'historicité de l'institution et l'évolution des formes d'écrit qui valident une formation est unique, nous ne l'avons trouvée qu'en Sciences de l'Education. A l'université de Lille3, lors de l'instauration du régime LMD (Licence, Master, Doctorat), suite aux accords de Bologne, et à la semestrialisation des études, les formations ont effectivement abandonné en première année de master le mémoire de maitrise et mis en place des «travaux d'étude et de recherche », plus courts, réalisables en un semestre. D'où cette opposition entre TER théorique et TER empirique, représentant les deux dimensions de l'ancien mémoire de maîtrise, découpé selon la logique semestrielle.

Ces différents discours permettent de cerner «le» mémoire de master, essentiellement en première année de master, par comparaison avec d'autres écrits universitaires ou en fonction de son évolution, en tenant compte de l'historicité des formations universitaires. Les enseignants considèrent le mémoire comme un genre d'écrit absolument particulier et cependant proche des autres (fiche de lecture, dissertation etc.). On peut supposer que pour eux, une des démarches d'apprentissage pourraient consister à faire prendre conscience des frontières communes et des spécificités irréductibles, même si la comparaison sur des dimensions communes à divers genres (le questionnement, la problématisation) est vraisemblablement très difficile.

\subsection{DIMENSIONS ÉPISTÉMOLOGIQUES}

Qu'en est-il des frontières qui sépareraient « les » mémoires entre eux, selon des critères, non plus textuels ou génériques, mais institutionnels ou disciplinaires? 


\subsubsection{Spécificités disciplinaires ou contextuelles?}

Les modes de définition des mémoires, dans le discours des enseignants, sont entièrement contextuels, liés aux contextes de formation, aux différentes institutions et à leur mode de fonctionnement, bien plus qu'aux contextes disciplinaires ou épistémologiques.

Ainsi les enseignants en Sciences du Langage évoquent en creux le mémoire professionnel, comme permettant d'identifier les spécificités du mémoire dit « de recherche ». C'est la seule discipline où cela est évoqué, mais avec beaucoup d'implicites, comme si c'était un objet partagé, ne nécessitant pas de définition. En Histoire, à Bruxelles, le mémoire de première année est présenté très explicitement et fréquemment comme une préparation à celui de deuxième année, et les transformations attendues entre les deux années sont souvent évoquées. C'est la dimension pédagogique de la formation à l'élaboration d'un travail de recherche qui alimente le discours des enseignants.

Ainsi, les disciplines universitaires ne sont pratiquement jamais évoquées, sauf dans deux cas très précis, qui vont être développés ci-après.

\subsubsection{Les disciplines de recherche des directeurs de memoire}

Les enseignants, quand ils réfléchissent aux dimensions disciplinaires du mémoire, s'appuient sur leurs disciplines de recherche, bien plus que sur les disciplines d'enseignement ou de formation.

C'est le cas de l'Histoire. Nous avons vu plus haut comment ces enseignants formulaient des attentes particulières à l'histoire contemporaine, qui déterminent, selon eux, ou des modes d'évaluation ou des manières de présenter l'objet d'une recherche, spécifiques à ce champ.

Les historiens de cette université belge vivent, en fait, selon une organisation institutionnelle en chaires, liées à un professeur et à sa spécialité

« on est séparé en chaires »

« on a l'impression qu'il y a là une espèce de tradition par séminaire

et par personne qui fait le séminaire » (Histoire, Bruxelles) 
Cette organisation produit des différences notables dans l'enseignement et une difficulté à définir des attentes progressives ou partageables. Chacun campe sur les évidences de sa spécialité.

Cette spécificité disciplinaire est également évoquée dans l'entretien mené en Sciences de l'Education. Dans cette discipline, telle qu'elle est enseignée à Lille, les mémoires sont relativement différenciés selon les parcours internes à la formation (Didactiques, Travail social, Travail éducatif, etc.). Ces parcours comportent une certaine dose d'interdisciplinarité (Sociologie de l'éducation, Histoire de l'éducation, Psychologie clinique, Didactiques, Analyse du travail éducatif, etc.) qui peut s'effacer lorsqu'entre en jeu la discipline spécifique du directeur de mémoire. Je retiens ci-dessous des extraits de l'échange entre un historien et une linguiste-didacticienne, sur la nature du mémoire de master, où l'on voit que les définitions de parcours internes aux Sciences de l'éducation (Histoire de l'Education, Didactiques, etc.) sont liées au sentiment, peu explicité, de différences de type épistémologique :

« - il y a une deuxième chose, la véracité des données théoriques, c'est-à-dire à partir du moment où il $\mathrm{y}$ a un sujet qui suppose des connaissances, la mise en route de connaissances, bon, il faut voir s'il y a des contre-sens énormes, alors d'autant plus que moi je suis historien, j'incite donc les étudiants à choisir des sujets qui aient au moins une dimension historique, même partielle, et donc à prendre au moins un vrai livre d'histoire parmi ceux qu'ils analysent, alors là je peux vérifier s'ils assimilent à peu près des données, des faits (JPM : 94)

«- enfin, nous on nomme les choses différemment, mais, en fait, ça rejoint ça, et en même temps je vois bien des critères spécifiques à ce que pourrait demander J.-P., c'est à dire à travers ce qu'il énonce, j'essaye d'imaginer le produit final qu'il attend, et il est sans doute différent de ce que nous on fait, mais voilà, c'est parce que c'est J.-P., parce que c'est un historien, on est bien en sciences de l'éducation mais je pense que le TER théorique produit par ses étudiants n'est pas le même que celui qui est produit par les étudiants en Didactique » (RH : 95) 
L'historien pose, entre autres caractéristiques du mémoire, la soumission à la véracité des faits historiques et reconnait orienter ses étudiants vers des mémoires ancrés dans cette discipline, ne serait-ce qu'un peu. Et la linguiste-didacticienne énonce la différence qu'elle entrevoit entre ses mémoires-là et ceux qu'elle dirige en didactique, où une des dimensions principales pour elle est celle de l'élaboration du questionnement de recherche et notamment la distance qu'arrive ou non à prendre l'étudiant par rapport à sa question de départ, qui est souvent le reflet d'idées de sens commun sur l'enseignement ou l'apprentissage.

« on accorde beaucoup d'importance à la question et à la construction de la question, ce qu'on appelle la problématique, mais j'aime pas beaucoup ce terme, c'est à dire qu'est-ce qu'on pose comme question? quelles sont les questions connexes qui tournent autour de cette question ? et comment on les traite ? et surtout aussi sur le dispositif méthodologique qui est envisagé, c'est-à-dire, pour répondre à cette question, qu'est-ce que je vais faire ? quel terrain j'investis ? quelles techniques j'emploie ? » (RH : 95).

Ces deux dimensions me semblent être le signe d'exigences disciplinaires différentes, dont le mémoire va être le lieu et le moyen d'une prise de conscience pour les étudiants les plus clairvoyants.

\subsubsection{L'écriture scientifique}

Par ailleurs, la référence du mémoire de master est, toujours pour certains de ces enseignants, l'écriture de recherche elle-même. Ce thème est développé par les historiens de Bruxelles et les enseignants en Sciences de l'Education. Voici d'abord un petit échange entre historiens :

« pour rejoindre ce que V. disait, c'est vrai que notre critère implicite c'est de se dire : «tiens, il est publiable » et donc notre modèle, c'est un bon article scientifique dans une bonne revue d'histoire (JMS : 73)

dès le début on leur demande ça (VP: 74)

et vous pourriez me le décrire parce que (Interviewer : 75) 
bien problématisé, rigoureux dans sa présentation, raisonnablement écrit avec une annotation ${ }^{15}$ qui se respecte, solide sans être excessive, avec une conclusion, et ça ne doit pas nécessairement être un énorme sujet, ça doit être novateur et former un tout bien cohérent, pourvu que le plan soit cohérent, qu'il y ait une bonne cohérence interne, ils font comme ils veulent » (JMS : 76)

Dans ce cas, le mémoire est construit comme un écrit semblable aux autres écrits «scientifiques », une production achevée...dont le contexte de production et de réception en formation universitaire n'a plus guère d'importance. Cette conception est particulièrement interrogeante pour l'enseignement qui accompagne la production de ce mémoire, puisqu'elle semble «interdire », disons pour le moins freiner, l'élaboration de dispositifs didactiques accompagnant l'écriture (LAHANIER-REUTER; DELCAMBRE, 2013).

Le second cas intéressant est celui des Sciences de l'éducation. Dans cette discipline, telle qu'elle est enseignée à Lille, le débat a porté également sur le rapport entre mémoire universitaire et écriture scientifique, du moins telle que les chercheurs peuvent la pratiquer dans certaines de leurs publications. Le discours ci-dessous est tenu par une spécialiste de Didactiques :

- «ça me rappelle comment on expose dans les revues anglaises, il y a quand même des formats incroyables, c'est à dire introduction, problématique, le cadre théorique, les résultats, les discussions, la conclusion, c'est hyper formaté, et je me souviens que j'avais une proposition de communication lors d'un colloque de psychologues et j'avais un peu fait un truc comme ça, introduction, méthode, résultats, et j'avais été acceptée, et quand j'ai débarqué dans ce lieu, j'étais la seule linguiste didacticienne et je me suis dit « qu'est-ce qui fait que je suis là » et en fait c'était la présentation de ma communication, ça collait parfaitement à certaines normes et c'est ça, donc il y a quelques fois un formatage de disposition qui fait que ça va passer là mais pas ici, que c'est propre à une discipline et pas à une autre, et moi je pense qu'il ne faut pas aller vers ça » (RH : 134)

15 Nous n'avons pas réussi à comprendre ce que veut dire ce terme d'annotations, probablement dû à une difficulté de transcription. 
- «Dans ce format-là, où serait l'évolution de la question de départ ? ça arriverait à quel moment et de quelle façon ?» $(\mathrm{RH}$ : 136)

On voit comment cette chercheure a, à la fois, l'expérience de l'organisation classique des écrits en Psychologie, dite IMRaD («Introduction, Methods, Results and Discussion»), et comment elle la juge inappropriée, par son formatage excessif, pour l'initiation à la recherche en éducation, notamment si l'on se donne l'objectif de la prise de distance par rapport à la question de départ.

L'écriture universitaire qui est indissociable des cadres scientifiques (ou des communautés de discours) dans lesquels et pour lesquels elle se développe est une des références, acceptée ou au contraire interrogée, des enseignants lorsqu'ils sont amenés à expliciter leurs attentes quant au mémoire de master.

Pour en terminer avec cela, on peut dire que le genre «mémoire » connait de multiples actualisations, selon les offres de formation, selon les organisations universitaires. C'est la comparaison avec des genres discursifs proches qui est la plus fréquente, les liens entre écriture du mémoire et épistémologie disciplinaire ou écriture scientifique sont établis par certains, mais cela reste épisodique. A l'issue de cette enquête, il est difficile d'affirmer que les écarts entre disciplines induisent «naturellement» (pour tous les enseignants) des différences entre mémoires. Il faut cependant avoir en mémoire que cette absence de précision dans leur discours vient probablement de la structure des entretiens de groupe réunissant des enseignants de la même discipline et de la même institution, structure qui est bien plus productrice de consensus implicite qu'une structure où les enseignants auraient été confrontés à des collègues d'autres disciplines et/ou d'autres institutions.

6 CONCLUSION

Cette étude a montré l'importance des ruptures entre les cursus Licence et Master dans les déclarations des étudiants: importance des modifications dans les pratiques des écrits, dans les représentations des 
normes et des attentes des enseignants. Ces ruptures justifient que les communautés universitaires considèrent l'écriture comme toujours en cours d'acquisition, y compris au niveau du Master. Contrairement aux idées communément répandues, la question de l'accompagnement de l'écriture des étudiants ne concerne pas que la première année d'université, sur le modèle le plus répandu aux USA. Les étudiants progressent dans leur trajet de la première à la cinquième année, comprenant lentement la spécificité de l'écriture universitaire, et ce d'autant plus qu'ils sont confrontés à des exigences de productions de recherche originales, proches de la production scientifique. Nos résultats militent donc contre les formes de remédiation pensées pour les seules premières années d'université, remédiations qui présentent le triple désavantage de faire porter sur l'enseignement secondaire le soupçon d'une incapacité à bien préparer les étudiants, de réduire l'accompagnement à des formes d'enseignement de compensation, portant sur des micro-habiletés linguistiques ou à des formes d'aide méthodologique, périphériques à la question de la maîtrise des genres discursifs, et enfin, de méconnaitre la nécessité de prodiguer aux étudiants un accompagnement de plus en plus spécifique au genre de discours requis et aux exigences disciplinaires.

A côté des ruptures, on constate aussi des continuités si l'on observe la lente transformation des normes personnelles et d'évaluation qui font passer les étudiants d'une conception linguistique de la production d'écrits aux représentations des normes d'un genre, l'écrit de recherche. Cette lente évolution montre a posteriori combien les expériences d'apprentissage des étudiants sont fécondes, y compris en ce qui concerne leurs représentations de l'écriture, y compris sans qu'ils aient reçu enseignement spécifique en la matière (DELCAMBRE; LAHANIER-REUTER, 2012).

Si l'écriture du mémoire de master s'inscrit dans une discipline donnée, l'importance de l'ancrage disciplinaire est un peu moins nette que celle des variations liées à la place dans le cursus, dont je viens de parler cidessus. Cela dit, les disciplines d'enseignement ou de recherche déterminent des pratiques différentes, sont des cadres qui pèsent sur les pratiques. Certaines de ces déterminations disciplinaires sont identifiables comme des traces de l'épistémologie des disciplines, du moins telle qu'elle 
est perçue par les étudiants (cf. les étudiants de Lettres et la dissertation, les deux enseignements d'Histoire différents qui se donnent à Lille et Bruxelles) et verbalisée par les enseignants (voir les discours en Sciences de l'Education et en Histoire). Mais cette inscription disciplinaire se fait parfois à l'insu de ceux qui enseignent et de ceux qui apprennent : elle n'est pas toujours très clairement exposée, que ce soit chez les étudiants ou chez les enseignants: peut-on expliquer ce flou dans les formulations par des problèmes de conscience disciplinaire, insuffisamment développée? Ou au rôle des implicites dans la communication? Ou encore à la structure des questionnements auxquels étaient soumis étudiants et enseignants, car on n'a interrogé frontalement et explicitement ni les étudiants ni les enseignants sur ces dimensions disciplinaires? La réduction de ces flottements pourrait être un objectif commun aux enseignants et aux étudiants, dans des structures où l'écriture serait un contenu disciplinaire au niveau master. Une des questions qui seraient ainsi à éclaircir pourrait être celle de l'élucidation des ruptures et des continuités entre l'écriture de recherche et l'écriture de recherche en formation (REUTER, 2004), dont on a vu qu'elle est inégalement partagée par les enseignants : certains, sûrs de leur légitimité, assimilant l'écriture des étudiants à la leur propre, d'autres au contraire interrogeant cette assimilation au nom de la formation et de la lente et nécessaire évolution des habitudes de pensée liée à la mise en œuvre d'une posture de chercheur.

\section{RÉFÉRENCES}

ADANGNIKOU, N. Peut-on parler de recherche en pédagogie universitaire, aujourd'hui, en France? Revue des Sciences de l'Education, XXXIV, 3, La recherche sur la pédagogie de l'enseignement supérieur. Où en sommes-nous?, p. 601-621, 2008.

BECQUET, V.; MUSSELIN, C. (2004). Variations autour du travail des universitaires. convention MENRT, ACI "Travail", Rapport CSO, Paris, 2004. $\mathrm{BOCH}, \mathrm{F}$. Linguistic analysis of Academic discourse. THe example of $\mathrm{PhD}$ students' Articles. Dans <http:://wac.colostate.edu/proceedings/wac2008/boch.pdf > (Éd.), Across Curricular Borders, WAC Conference. Austin, Texas, 2008. 
BOCH, F., LABORDE-MILAA, I.; REUTER, Y. (2004). Pratiques, n. 121-122, Les écrits universitaires.

CHEVALLARD, Y. La Transposition didactique : du savoir savant au savoir enseigné. Grenoble: La pensée sauvage, 1991.

DAUNAY, B. The evolution of the French Field of Didactique de l'écrit:

Theorizing the Teaching Practices of Writing in the Discipline. Dans REUTER, Y.; DONAHUE, C. (Dir.), Disciplines, Language, Activities, Cultures: Perspectives on Teaching and Learning in Higher Education From France and The United States. Amsterdam: Kluwer Academic Publishers, 2008.

DAUNAY, B.; LAHANIER-REUTER, D. Students' discourse on ease and difficulty in writing: A specific approach to the genres of academic writing. Dans DELCAMBRE, I.; LAHANIER-REUTER, D. International studies on writing at university: comparisons and evolutions. Fort Collins, Colorado: The WAC Clearinghouse/Parlor Press. (À par.)

DE KETELE, J.-M. La pédagogie universitaire: un courant en plein développement. Revue Française de Pédagogie, n. 172, p. 5-13, 2010.

DELCAMBRE, I. Discours d'autrui et littéracies universitaires. Didactiques, n. 2, Actes du $2^{\circ}$ colloque international sur la sémiotique, la didactique et la communication de Medea Algérie, 2012, p. 5-16.

DELCAMBRE, I.; BOCH, F. Normes et styles de l'écriture universitaire, une question d'apprentissage. Le Mook Autrement, Apprendre et transmettre, 2011. p. 104-109.

DELCAMBRE, I.; LAHANIER-REUTER, D. Ecrits et disciplines dans l'université française: le cas des sciences humaines. Dans DEFAYS, J.-M.; ENGLEBERT, A.; POLLET, M.-C.; L. ROSIER, L.; THYRION, F. Acteurs et contextes des discours universitaires. Paris: L'Harmattan, 2009. p. 151-166.

. Continuités et ruptures dans les pratiques d'écriture à l'université:

l'exemple de cursus en Sciences Humaines. Bulletin Suisse de Linguistique Appliquée, v.1, n. 93 (Les compétences langagières dans la formation et dans la vie professionnelle. Transitions et transformations), p. 115-130, 2011.

. De quelques pratiques universitaires de littéracie. Discours croisés d'étudiants et d'enseignants sur l'écriture en Sciences de l'Education. Les Cahiers $d u$ Cerfee, n. 30, p. 53-74, 2011.

. Difficultés de l'écriture académique en Sciences Humaines et perceptions de l'accompagnement: analyse de discours d'étudiants. Diptyque, n. 24 (De la maîtrise du français aux littéracies dans l'enseignement supérieur), p. 37-61, 2012. 2012. . Pratiques, n. 153-154, Littéracies universitaires: nouvelles perspectives, 
DELFORCE, B. Les difficultés langagières: pour une autre hypothèse explicative. Recherches en Didactiques, n. 12, p. 145-170, 1982/2011.

. Approches didactiques de la production d'un écrit "fonctionnel": les difficultés de la dissertation. Pratiques, n. 48, Les écrits non-fictionnels, p. 35-52, 1985.

DENIZOT, N.; MABILON-BONFILS, B. La dissertation: déclinaisons disciplinaires d'un objet scolaire. Recherches en Didactiques, n. 14, Activités disciplinaires dans le secondaire, p. 11-27, 2012.

ENDRIZZI, L. Savoir enseigner dans le supérieur: un enjeu d'excellence pédagogique. 2011. Consulté le octobre 25, 2012, sur inrp.fr/vst:

$<$ http://www.inrp.fr/vst/DA/detailsDossier.php?dossier=64\&lang=fr $>$

FABIANI, J.-L. A quoi sert la notion de discipline? Dans BOUTIER, J.;

PASSERON, J.C.; REVEL, J. (Eds.), Qu'est-ce qu'une discipline? Paris: Ecole des Hautes Etudes en Sciences Sociales, 2006. p. 11-34.

. Du chaos des disciplines à la fin de l'ordre disciplinaire? Pratiques, $\mathrm{n}^{\circ} 153-$ 154, p. 129-140, 2012

LAHANIER-REUTER, D.; DELCAMBRE, I. Le mémoire de master dans le discours d'enseignants universitaires: entre travail de recherche et écriture académique. Dans ROMAINVILLE, M.; FRENAY, M. L'accompagnement des mémoires et des thèses, Actes des XII ${ }^{\circ}$ rencontres du REF, Louvain-la-Neuve, sept. 2011. Louvain-la-Neuve: Presses universitaires de Louvain, 2013. p. 129-152.

REUTER, Y. De quelques obstacles à l'écriture de recherche. Lidil, n. 17, p. 11-23, 1998.

. Analyser les problèmes de l'écriture de recherche en formation. Pratiques, n. 121-122, p. 9-27, 2004.

. La conscience disciplinaire. Présentation d'un concept. Education et Didactique, v. 1, n. 2, p. 57-71, 2007.

. Dictionnaire des concepts fondamentaux des didactiques. Bruxelles: De Boeck Université, 2007/2010.

SAUSSEZ, F.; LOIOLA, F. A. Les conceptions des professeurs d'université à propos de l'enseignement, sous la lunette de la psychologie culturelle. D'une analyse critique des écrits à des perspectives pour la recherche. Revue des Sciences de l'éducation, XXXIV, 3, La recherche sur la pédagogie de l'enseignement supérieur. Où en sommes-nous?, p. 569-599, 2008.

ANNEXES 
ANNEXE 1 - Les écrits pratiques selon les étudiants (master vs licence)

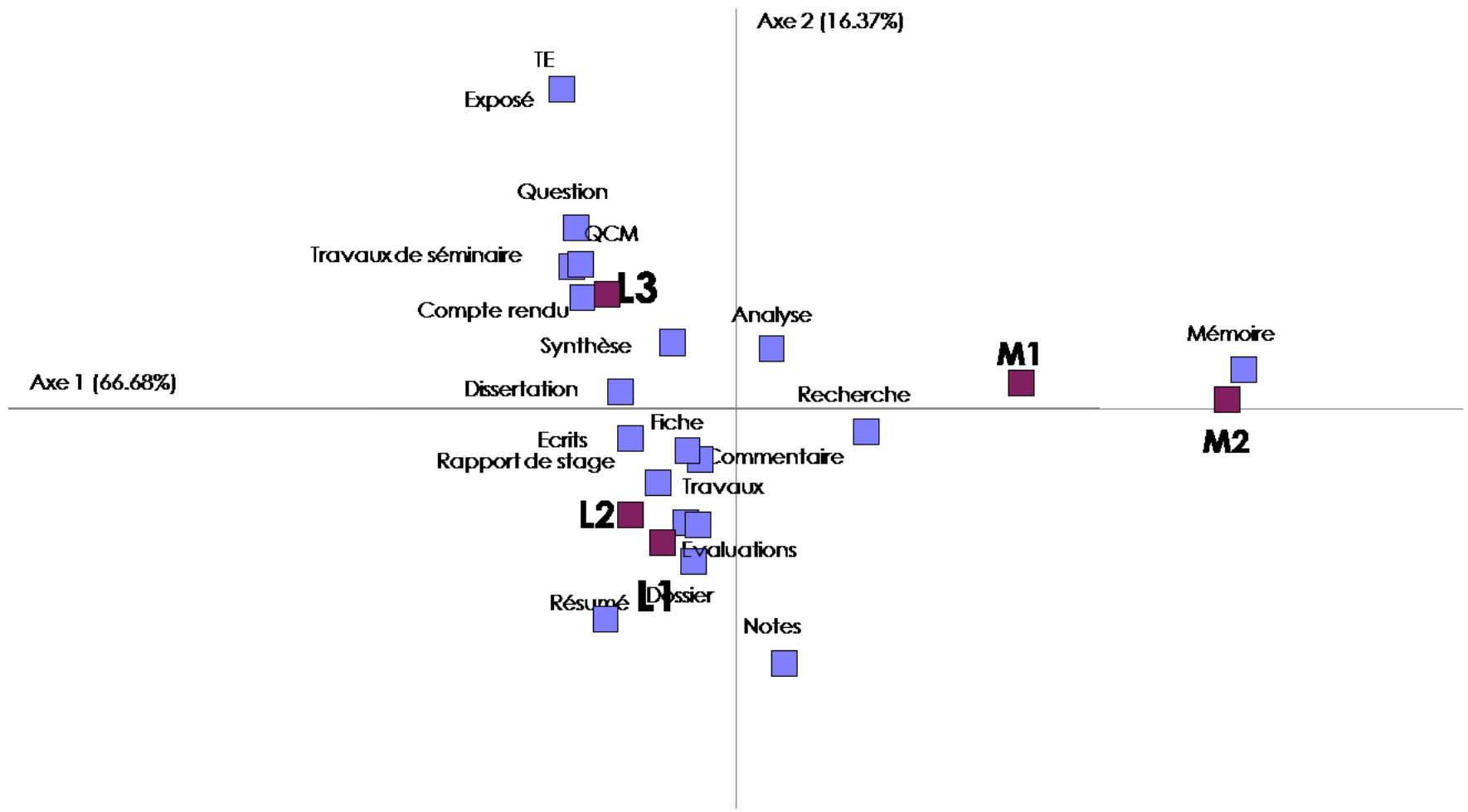


ANNEXE 2 - Que dissent les étudiants de leurs normes personelles?

(master vs licence)

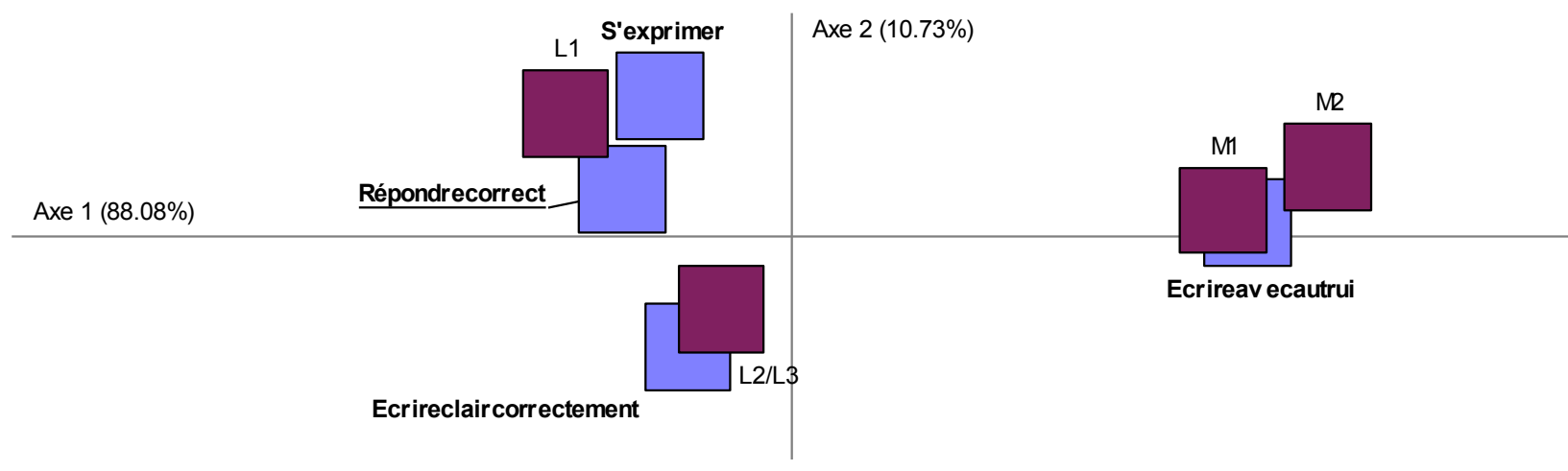


ANNEXE 3 - Que disent les étudiants des attentes des enseignants? (master vs licence)

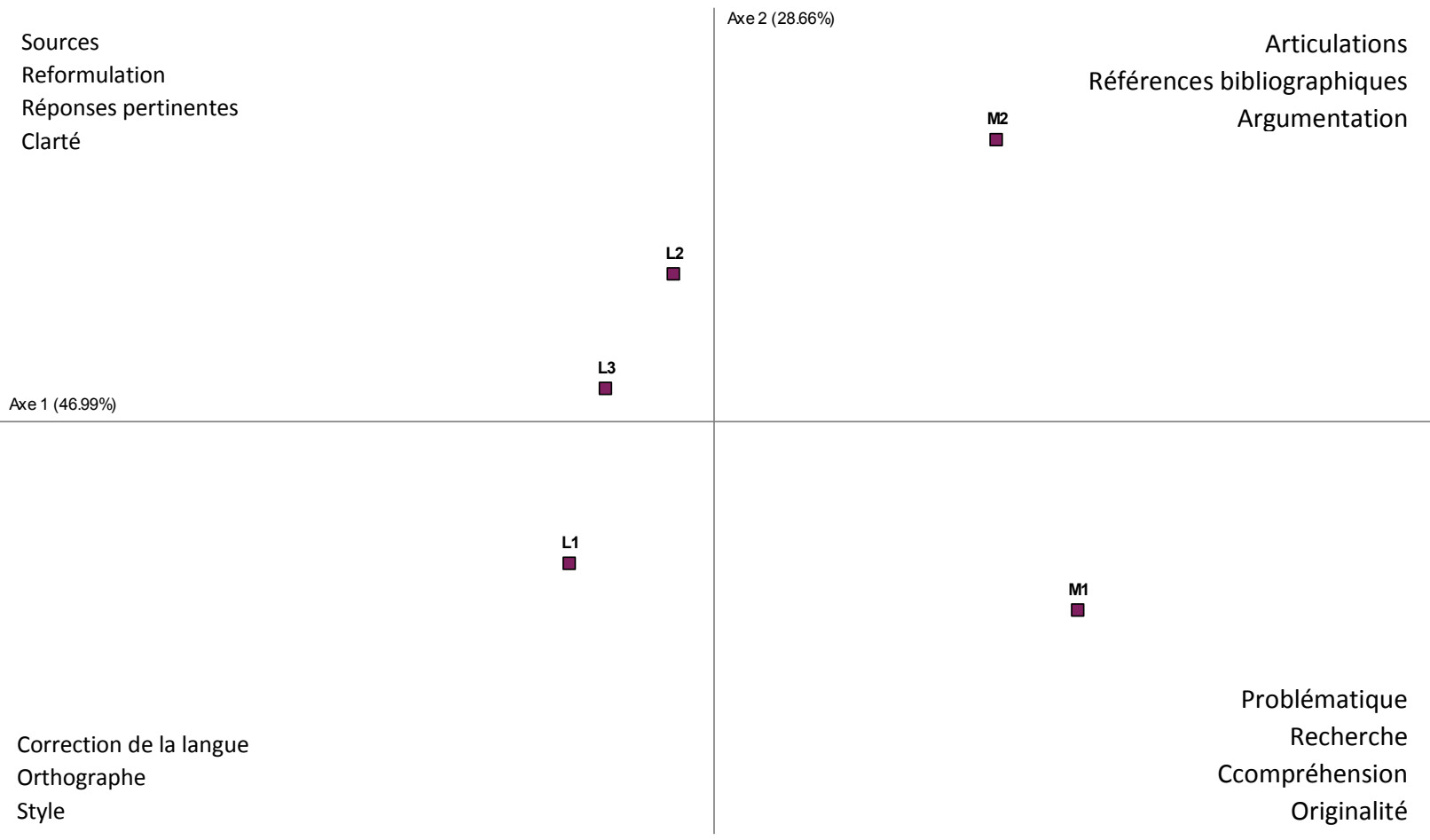


ANNEXE 4 - Genres d' écrits et difficultés déclarées

AXE $2(32.26 \%)$

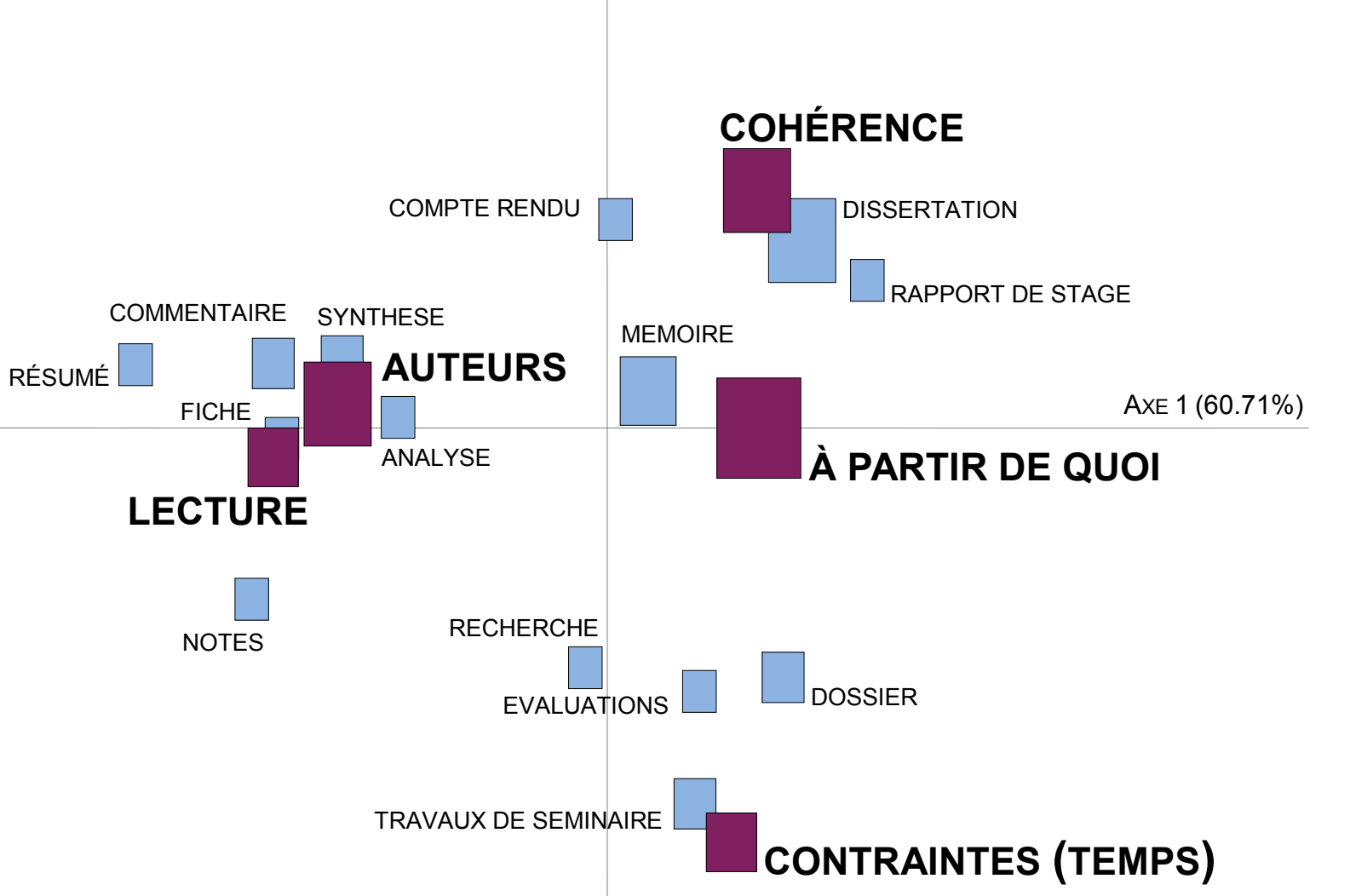


ANNEXE 5 - Les Normes Personnelles Selon Les Disciplines

\begin{tabular}{|c|c|c|c|c|}
\hline & Axe 2 (24.56\%) \\
Lettres \\
Modernes $1(70.12 \%)$
\end{tabular}


ANNEXE 6 - Les normes d' évaluation selon les disciplines

A PARTIR DE QUOI

$\begin{array}{rr}\text { Axe 2(23.31\%) } & \text { Sources } \\ & \text { Référénces Bibliographiques }\end{array}$

Histoire Lille

口

Sciences du langage

$\begin{array}{ccc}\text { Axe } 1(35.69 \%) & \text { Histoire Sciences de l'éducation } \\ & \square & \\ & & \\ & \text { Psychologie } \\ & & \\ & & \text { Lettres Modernes }\end{array}$

AUTEURS

Linguagem em (Dis)curso, Tubarão, SC, v. 13, n. 3, p. 569-612, set./dez. 2013. 
ANNEXE 7 - Les types de difficultés selon la discipline

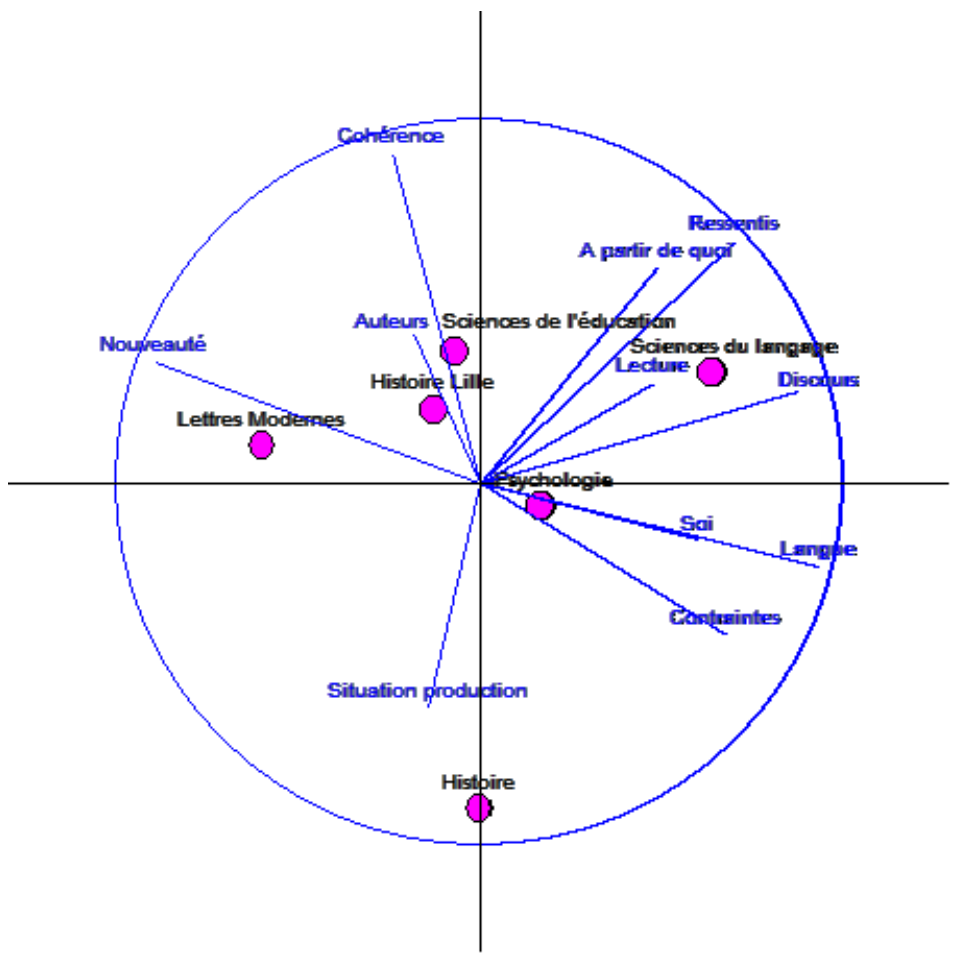

Recebido em: 04/06/13. Aprovado em 03/12/13. 
Title: The master thesis: ruptures and continuities. Professors' views, students'views

Author: Isabelle Delcambre

Abstract: Writing is a fundamental component of all courses in higher education; it is requested in all devices whose objectives come in terms of teaching, learning and training, from the first year of university through PhD. At Masters level, students and teachers are faced with a new dimension of academic writing: the production of scientific knowledge, insertion into a research field. Thereby, the master thesis presents for students a new subject relative to writings they have previously performed, new challenges related to the specific dimensions of writing during research training. As for professors, the accompaniment of these new writings is linked to specific expectations, strongly linked to different disciplinary epistemologies. This paper aims to understand and analyze issues of these writings in university education and the ways teachers and students comprehend them.

Keywords : University Literacy. Didactics. Disciplines. Curriculum.

Titulo: La disertación de maestría: rupturas y continuidades. Puntos de vista de los profesores, puntos de vista de los estudiantes

Autor: Isabelle Delcambre

Resumen: La escritura es un componente fundamental de todas las formaciones de la enseñanza superior; ella es solicitada en todos los dispositivos cuyos objetivos se declinan en términos de enseñanza, de aprendizajes y de formación, desde el primer año de la universidad hasta el doctorado. En el nivel de maestría, estudiantes $y$ profesores son confrontados con una nueva dimensión de la escritura universitaria: la producción de conocimientos científicos, la inserción en un campo de pesquisa. La disertación de maestría presenta, así, para los estudiantes una novedad relativa a la escritura que ellos practicaron anteriormente, nuevas dificultades ligadas a las dimensiones específicas de la escritura de investigación en formación, y para los profesores, el acompañamiento de esos nuevos escritos dice respecto a expectativas específicas, fuertemente ligadas a las diferentes epistemologías disciplinares. Este artículo visará comprender las apuestas de esos escritos en la formación universitaria y el modo como profesores y estudiantes las aprenden.

Palabras-clave: Instrucción universitaria. Didácticas. Disciplinas. Curriculum. 
Título: A dissertação de mestrado: rupturas e continuidades. Pontos de vista dos docentes, pontos de vista dos estudantes

Autora: Isabelle Delcambre

Resumo: A escrita é um componente fundamental de todas as formações do ensino superior; ela é solicitada em todos os dispositivos cujos objetivos se declinam em termos de ensino, de aprendizagens e de formação, desde o primeiro ano da universidade até o doutorado. No nível de mestrado, estudantes e professores são confrontados com uma nova dimensão da escrita universitária: a produção de conhecimentos científicos, a inserção num campo de pesquisa. A dissertação de mestrado apresenta, assim, para os estudantes uma novidade relativamente à escrita que eles praticaram anteriormente, novas dificuldades ligadas às dimensões especificas da escrita de pesquisa em formação, e para os professores, o acompanhamento desses novos escritos diz respeito a expectativas especificas, fortemente ligadas às diferentes epistemologias disciplinares. Este artigo visará a compreender as apostas desses escritos na formação universitária e o modo como professores e estudantes as apreendem.

Palavras-chave: Letramento universitário. Didáticas. Disciplinas. Currículo. 